\title{
Pedicle subtraction osteotomies (PSO) in the lumbar spine for sagittal deformities
}

\author{
Pedro Berjano $\cdot$ Max Aebi
}

Received: 24 October 2014/Revised: 5 November 2014/ Accepted: 5 November 2014/Published online: 9 December 2014

(C) The Author(s) 2014. This article is published with open access at Springerlink.com

\begin{abstract}
Introduction The narrow correlation between sagittal alignment parameters and clinical outcomes has been widely established, demonstrating that improper sagittal alignment is a clinical condition that is associated with increased pain and limitations in patients' functional ability.

Indication Lumbar pedicle subtraction osteotomy (PSO) is indicated in the treatment of large sagittal (more than $25^{\circ}$ of rigid loss of lordosis) deformities of the lumbar spine or its combination with coronal deformity, especially when they are rigid. Indication should be based on careful assessment of the severity of symptoms, functional impairment, functional expectations of the patient, general clinical condition and surgical and anesthesiological team experience. Risk should be carefully assessed and discussed to obtain appropriate informed consent.

Surgical procedure Surgical planning includes selection of the safest levels for the upper and lower instrumented vertebra, site of the osteotomy, modality of fixation, and, most importantly angular value of the correction goal (target lumbar lordosis). Failure to adequately obtain the necessary amount of sagittal correction is the most frequent cause of failure and reoperation.

Conclusion PSO is a valuable surgical procedure in correction of severe hypolordosis (=relative kyphosis) in the
\end{abstract}

\author{
P. Berjano \\ IRCCS Istituto Ortopedico Galeazzi, Milan, Italy \\ M. Aebi \\ University of Bern $(\mathrm{CH})$ and McGill University, \\ Montreal, Canada \\ M. Aebi $(\bowtie)$ \\ "Das Rückenzentrum", Hirslanden-Salem Hospital, \\ Schänzlistrasse 39, 3000 Bern 25, Switzerland \\ e-mail: max.aebi@MEMcenter.unibe.ch
}

lumbar spine. It is a demanding procedure for the surgeon, the anesthesiologist and the intensive care team. Although its complication rate is high, it has a substantial positive impact in the quality of life of patients, including the elderly.

Keywords Pedicle subtraction osteotomy · Sagittal imbalance · Adult deformity - Lumbar kyphosis - Lumbar spine

\section{Introduction}

The narrow correlation between sagittal alignment parameters and clinical outcomes has been widely established in the last years, demonstrating that improper sagittal alignment is a clinical condition that is associated with increased pain and limitations in patients' functional ability [1-3]. Recent classifications [4,5] of adult deformity take into account sagittal alignment as a major factor determining outcomes. Recently, a validated, comprehensive classification of sagittal deformities has improved the comprehension of the deformity, showing how sagittal misalignment in any region of the spine has an influence on the rest of the spine, with reciprocal compensatory changes that express as deformity patterns (regional deformity plus typical compensatory changes for each type of deformity are a deformity pattern), and assisting the surgical planning [6].

Three major metric parameters that account for a better result have been extensively validated: lumbar lordosis/ pelvic incidence mismatch (LL-PI), pelvic tilt (PT) and sagittal vertical axis (SVA) [7, 8]. The first of these parameters (PI) is actually the only morphologic indicator of alignment and its advantage is that it can be measured independently of the patient's position (i.e., in the surgical decubitus position); the other parameters are positional 
parameters that present themselves as the result of the interaction between spinopelvic morphology (PI-LL, and thoracic kyphosis-TK-mainly) and muscular activity, and can only be measured with the patient standing in a full lateral spine film [9]. Recent research has provided reliable methods to predict the positional parameters PT and SVA from patient's age and morphological parameters (PI, LL, TK), thus permitting a calculation of the adequacy of alignment with the patient in decubitus. This finding is of paramount importance to intraoperatively predict the result of surgery in terms of alignment [10]. Different surgical maneuvers can be used to correct abnormal sagittal alignment. Minor amounts of PI-LL mismatch can be surgically addressed with standard surgical maneuvers (cage positioning, partial arthrectomy, posterior compression, rod contouring), and generally these maneuvers can increase the lumbar lordosis by $5^{\circ}-7^{\circ}$ per instrumented level. In some instances of collapsing spine (a major PI-LL mismatch is present and the spine is still flexible in traction or fulcrum-extension films), these methods can also provide correct alignment. Conversely [11], in non-flexible spines with a major LL-PI mismatch, greater corrections are needed and osteotomies of the spine are the most frequently used method of correction. Posterior column osteotomies $[12,13]$ have a more limited power of correction, in the range of $8^{\circ}-10^{\circ}$ per osteotomy level. The combination of large anterior cages with posterior column shortening osteotomies can provide a greater correction $\left(10^{\circ}-15^{\circ}\right.$ per level). Tricolumnar pedicle subtraction osteotomies (PSO) give a greater correction at a single point of the spine, ranging from $20^{\circ}$ to $35^{\circ}$ per level.

Though both lumbar lordosis and thoracic kyphosis have an influence on final alignment, it has been demonstrated [14] that it is the amount of lumbar lordosis in relation to the pelvic incidence that is the most influential parameter for the prediction of the final standing alignment of the spine, being the amount of thoracic kyphosis less critical regarding the final alignment of the patient.

For this reason, PSO in the lumbar spine is one of the most frequent procedures in the treatment of severe sagittal misalignment of the spine in adults.

In this article, we will discuss the indications, decision rules and surgical technique of PSO in the lumbar spine for sagittal deformities.

\section{Indications}

Lumbar PSO is indicated in the treatment of sagittal or sagittal and coronal deformity of the spine in presence of hypolordosis, spine stiffness (full correction cannot be achieved in fulcrum extension radiographs) and when the amount of necessary correction of LL is large $\left(25^{\circ}\right.$ or more,
Fig. 1). The decision to operate on an individual should take into account the severity of symptoms, the progression of the deformity and the clinical condition of the patient, as tricolumnar osteotomies of the spine carry substantial risk of complications. Also the expectations and functional needs of the patients should be considered and discussed, as the long fusions of the spine frequently needed for the correction of sagittal deformities in the lumbar spine determine a permanent functional limitation in patients. Whereas the elderly patients have the highest risk of complications associated to this surgery, they are the subgroup of patients who are more likely to obtain significant benefit from correction of the deformity, as has been shown by Smith et al. [15]. A complete surgical evaluation should include long-lateral standing films of the spine including the cervical spine and the hip joints in the same image and lateral fulcrum-hyperextension film in supine position, with the bolster at the level of the maximum deformity. From the standing full-spine film the necessary correction can be calculated based on geometrical methods [16, 17] (basically consisting in measuring the target angle of the osteotomy as a combination of the angular translation of C7 and the change in pelvic version required). Similarly, nongeometrical methods of calculation of the required correction can be used. These are based on the experimental verification for a given pelvic incidence, and a given combination of LL and TK, which causes a correct standing alignment [18]. If no other deformities are present (i.e., kyphosis in the thoracolumbar transition), the target lumbar lordosis can simply be calculated by adding $10^{\circ}$ to the given PI in this specific patient [14]. A more exact nongeometrical calculation can be made based on the relationship between PI and LL experimentally found in asymptomatic volunteers [19]. Some of these methods lack a calculation of the potential increase in thoracic kyphosis after surgery (especially in patients with compensatory thoracic hypokyphosis in whom the thoracic spine is not included in the fusion area) and the surgeon should take the TK change into account, as it could result in final hypocorrection of SVA/PT. When correctly used, both geometrical and non-geometrical methods of calculation of the necessary correction can provide satisfactory results (Table 1).

The choice of level for the osteotomy depends on several factors. When a sagittal angular deformity is present, the osteotomy should ideally be performed at the apex of the sagittal deformity. Another relevant factor is the convenience of restoring the normal shape of the spine, where $60 \%$ of the lordosis is located between L4 and S1. Most patients have the largest lack of lordosis in the lower lumbar spine, and for this reason the authors most frequently perform the PSO at L4. This permits having three levels of fixation below (L5, S1 and the ilium) (Fig. 2). An 

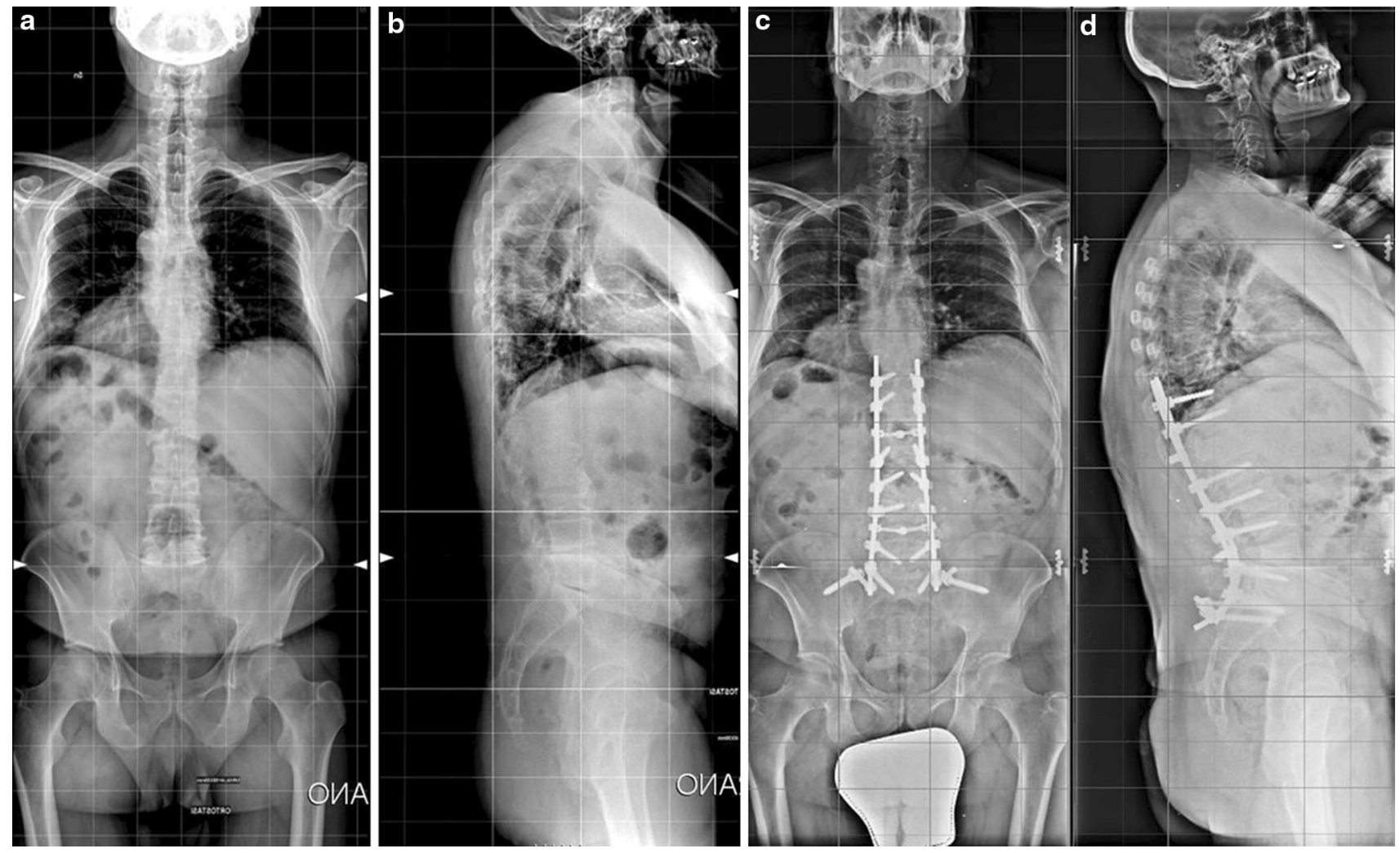

Fig. 1 Preoperative (a, b) and postoperative (c, d) X-rays of a 47-year-old male treated with a T10-pelvis fusion and L4 PSO for a lumbar hypolordosis. It can be noticed how the lumbar lordosis changed from $20^{\circ}$ preoperatively to $45^{\circ}$ postoperatively

Table 1 Indications for PSO

\section{Indications for PSO}

Objective (measurable: X-rays, other imaging)

\section{Criteria}

Subjective (history and clinical findings)
- Proven progression of the curve

- Sagittal or sagittal and coronal deformity

- Presence of lumbar hypolordosis

- Spine stiffness in functional X-rays (supine position in extension over fulcrum, traction films)

- If amount of correction needed is $\geq 25^{\circ}$

- Amount of thoracic kyphosis when stiff or flexible

- Osteoporosis (bone density measurement), old fractures, pending new fractures, etc.

- Spinal canal narrowing: central, recesal, foraminal (MRI and/or Myelo-CT)

- Severity of symptoms

Back pain (when upright, walking, in bed, with physical activity)

Radicular pain (constant, only when upright standing, or when sitting or lying)

Claudication symptomatology: walking distance

- Combined with neurological signs

Sensory disturbances, motor deficit, pos. Lasègue sign

- Clinical balance: amount of leaning forward: need for rollator or crutches

- Morbidity status (pulmonary, cardiac, renal thromboembolic disorder, allergies, overweight, previous spine and other surgery

- Expectations of the patient 


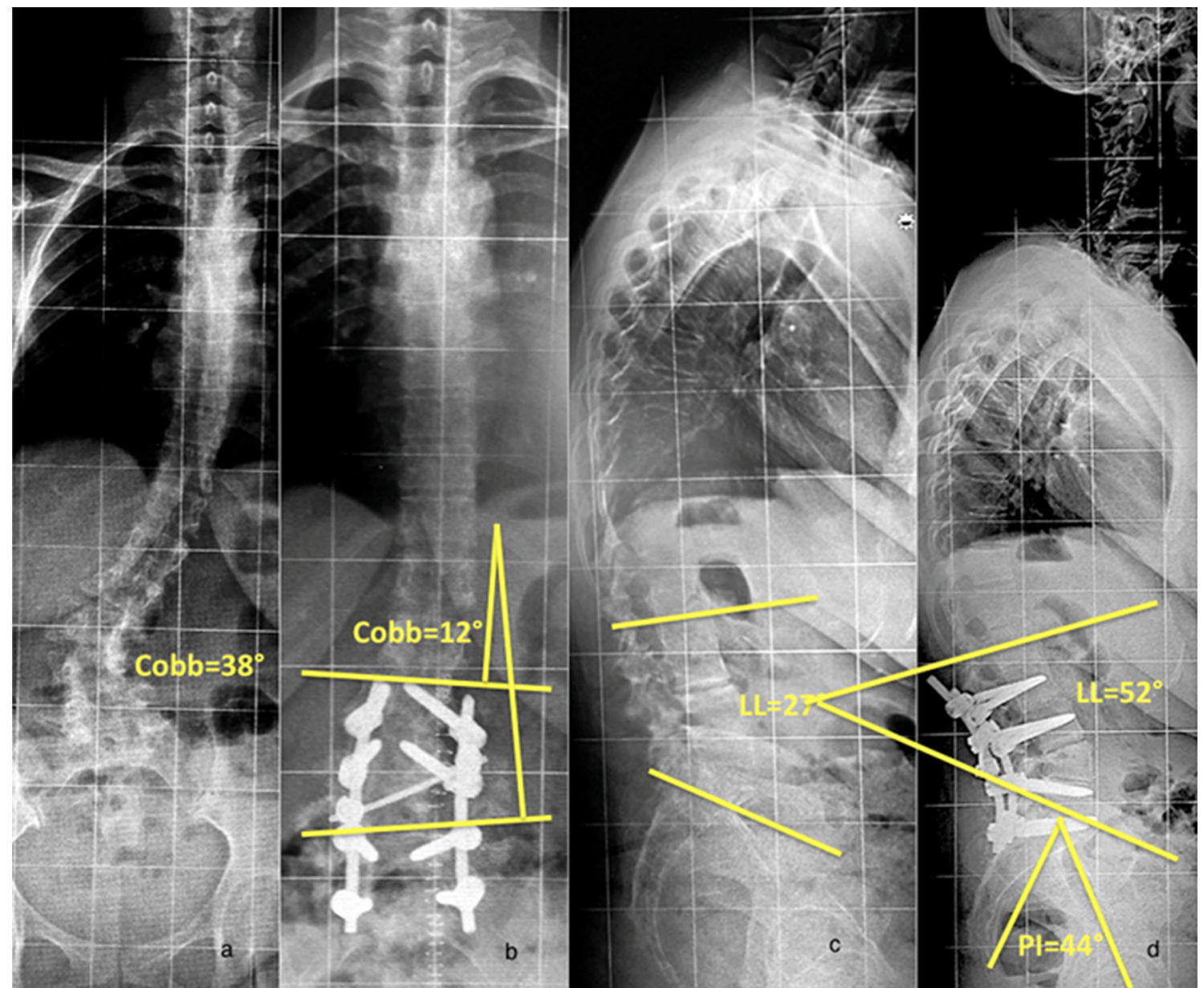

Fig. 2 Preoperative $(\mathbf{a}, \mathbf{c})$ and postoperative $(\mathbf{b}, \mathbf{d}) \mathrm{X}$-rays of a 53-year-old female patient with severe low back pain and pain with bilateral leg irradiation. A secondary adult lumbar degenerative scoliosis with spondylolysis and-listhesis grade I at L5/S1 with root irritation at L5 and L4 (LL $27^{\circ}$, PI $44^{\circ}$ ) was observed. A short lumbar fusion from L2 to S1 with a L4 PSO was performed, to restore an adequate lumbar lordosis $\left(52^{\circ}\right)$ in relation to PI

degenerative conditions the most frequent fusion areas are L2-S1 (Fig. 2), T10-S1 (Fig. 1) and T2-S1 (Fig. 5). Stopping the fusion proximally at the thoracolumbar junction or near the apex of the thoracic kyphosis, is usually not recommended, as it may result in increased incidence of proximal junctional kyphosis (PJK). This is especially true in case of a concomitant osteoporosis. Fusion to the higher thoracic spine is usually necessary in deformities with high SVA (more than 10-15 cm), in the presence of Parkinson's disease [21, 22], osteoporosis and in patients who do not compensate with active extension of the thoracic spine [11] (Table 2).

\section{Surgical technique}

The patient is placed in prone position on a surgical table that allows for appropriate padding (as the duration of surgery can result in decubitus lesions), abdominal 
Table 2 Location of the PSO and extent of fixation for correction and its maintenance

\begin{tabular}{c}
\hline $\begin{array}{c}\text { Location of the } \\
\text { OT }\end{array}$ \\
inciple: at the level of the apex of the kyphosis or \\
(best effect since $60 \%$ of lordosis is between L4 \\
and S1) \\
$\begin{array}{c}\text { Extension of } \\
\text { Pixation }\end{array}$ \\
at L3 fixation min. L1-L5 or OT at L4, fixation \\
min. L2-sacrum/pelvis \\
Above or below, but not at the TH-L junction: \\
L2-S1/pelvis or TH 10-S1/pelvis \\
Never stop at the apex of the thoracic kyphosis \\
(Th4-8): end at Th2-S1/pelvis \\
In rigid thoracic kyphosis without the potential of \\
compensation: Th2-S1 (plus rigorous posterior \\
release) \\
SVA $>6$ cm: long fixation necessary \\
Parkinson disease and malalignment: long fixation \\
In osteoporosis do not stop at thoracic kyphosis \\
(prophylactic vertebral body augmentation: end \\
vertebra with screws and two vertebra above)
\end{tabular}

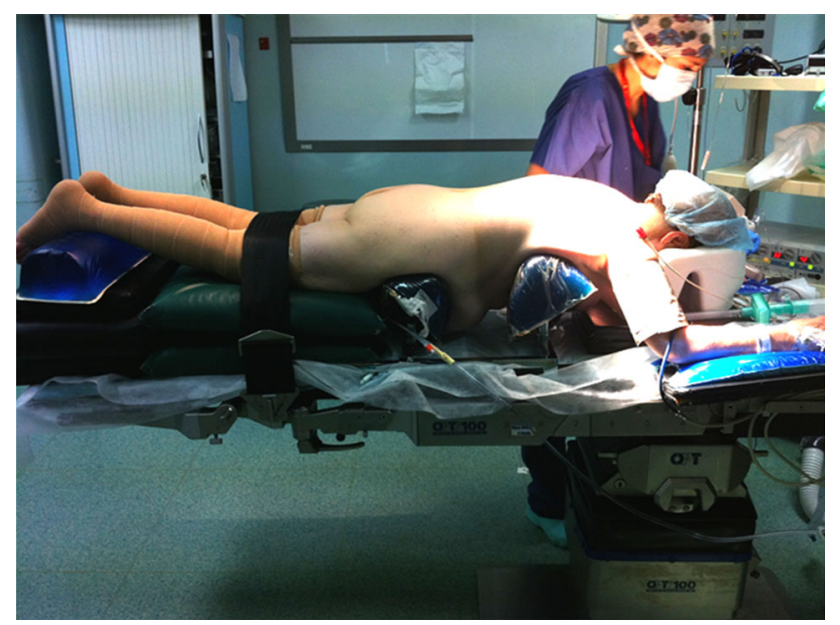

Fig. 3 Patient's positioning with face and eye protection, appropriate padding, abdominal decompression and full extension of the hips. Arms are in a neutral position with less than $90^{\circ}$ of shoulder abduction, antepulsion of the arms, less than $90^{\circ}$ of elbow flexion

decompression to prevent excessive epidural bleeding and reverse table bending to provide sagittal correction after the osteotomy, prior to instrumentation. Full extension of the hips is achieved with cushions under the thighs to increase the ability of correcting the sagittal profile by table reverse break (Fig. 3).

Preoperative prophylactic antibiotics are given. Meticulous care is taken in sterility and skin preparation. Appropriate actions to prevent loss of temperature are necessary to improve the fluid management and prevent hypocoagulability of the patient. When necessary, recording of multimodal evoked potentials is prepared. Exposure and dissection are made subperiosteally and sequentially to reduce the time that the spine is fully exposed, thus reducing blood loss, and heat and fluid loss by evaporation in the exposed field. Frequent irrigation of the field is performed to reduce the bacterial load in the wound. Instrumentation, usually by pedicle screw fixation and iliac bolts is performed first. The level where the osteotomy will be performed is left without any instrumentation. Some surgeons prefer topping off with sublaminar bands or hooks in the most cranial instrumented level or levels to create a progressive transition from the tricolumnar stiffness provided by pedicle screws to the mobile spine above the instrumentation and reduce the risk of PJK. Other strategies to reduce the risk of PJK include cement vertebral augmentation of the 1-2 levels above the instrumentation (Fig. 4) and avoiding any corrective forces on the upper two instrumented levels. After completion of the instrumentation, facetectomies and a rigorous posterior release are performed at every level to increase the flexibility of the spine. Once the exposure and instrumentation are complete, and meticulous hemostasis has been achieved, the osteotomy is begun. Generally all the posterior elements from $1 \mathrm{~cm}$ below the pedicle screws of the vertebra above the osteotomy site to $1 \mathrm{~cm}$ above the pedicle screws of the vertebra below the osteotomy site have to be resected. In addition, the spinous processes of the two adjacent vertebrae are completely resected. Additional bone and ligament is resected as needed to expose the exiting roots above and at the osteotomy level. Hemostasis with bipolar coagulation and hemostatic agents is performed at every step to reduce the blood loss during the procedure. To reduce the chance of pseudarthrosis around the osteotomy, interbody fusion at the discs above and below the osteotomy is suggested (Fig. 5). In case it is performed posteriorly with PLIF or TLIF, they should be made at this moment. When an anterior (ALIF or LLIF) approach is chosen, it can be performed before the posterior procedure (to minimize blood loss and fracture of the osteotomized vertebra, as could be the case when the anterior procedure is performed after the vertebra has been weakened by the PSO). The next step is osteotomy of the base of the transverse process at the osteotomy level. It can be performed with an osteotome or a Kerrison rongeur. Then, careful dissection of the lateral wall of the vertebral body is performed in posterior to anterior direction with a small Cobb retractor, avoiding to extend the dissection too far caudal (to prevent damage to the segmental vessels). After exposure of the lateral wall, the pedicle is resected to its base with a rongeur. At this point, the vertebral body osteotomy can be performed. The most frequently used technique is decancellation. A pedicle probe is passed into the vertebral body bilaterally to initiate decancellation. Increasing size probes or increasing size bone taps (up to 


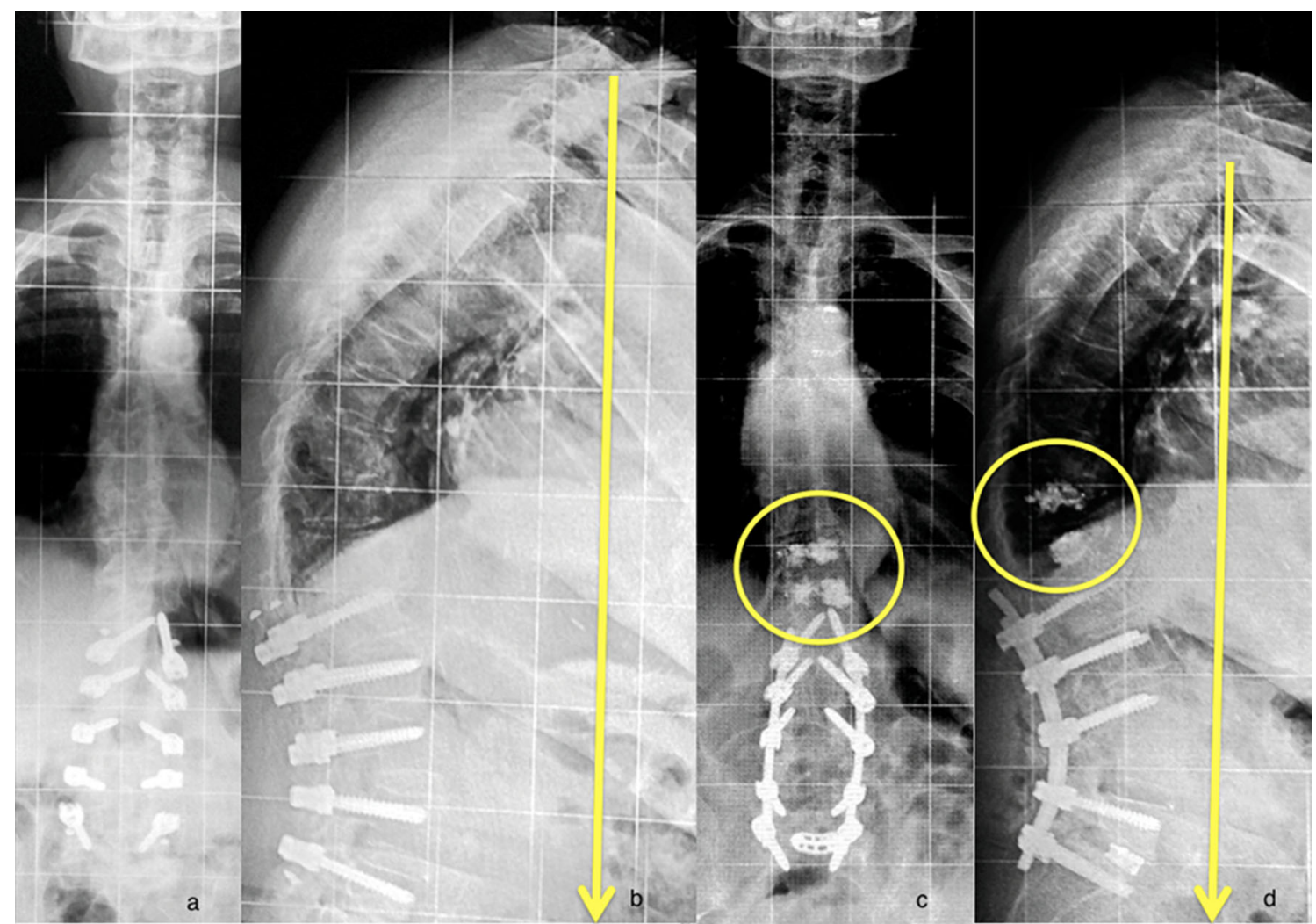

Fig. 4 a, b Preoperative A and lateral X-rays of a 82 year-old female patient with severe back pain (minimal leg pain) and clinically severe leaning forward posture, with a previous dynamic fixation from L1 to L5. Due to the age of the patient and significant polymorbidity and overweight, it has been decided to limit the surgery to the lumbar spine and the thoracolumbar junction $(\mathbf{c}, \mathbf{d})$, applying a rigid fixation from L1 to S1 including a L5/S1 circumferential fusion with TLIF, PSO at L4 and prophylactic cement augmentation of Th12 and Th11 to prevent secondary fracture and junctional kyphosis

8-9 $\mathrm{mm}$ ) are inserted in sequence to enlarge the perforations into cancellous bone. After this, curettes are used to increase osteotomy medially, laterally, cranially and caudally. Care is taken to create a posterior-based triangular wedge. Excessive decancellation in height in the anterior part of the vertebral body can result in parallel collapse of the vertebra with significant loss of the ability to provide sagittal correction.

After decancellation has been completed in the posterior aspect of the vertebra extending from lateral to lateral wall, retraction of the dural sac medially permits exposure of the posterior wall, which can be resected with Kerrisons. The most medial, thinned posterior wall can then be impacted anteriorly under the dural sac with angled bone tamps. After doing this, resection of the lateral walls is performed to complete the osteotomy [23]. Several maneuvers have been described to close the osteotomy. The authors' preferred method is reverse breaking of the table, as it does not put any stress on the instrumentation. Other methods include a cantilever maneuver with the contoured rod (in this case the rod should be solidly fixed at least to three solid anchors below the osteotomy and pushed to the anchors above the osteotomy). Repeated contouring of the rod, extremely sharp contouring or use of rod benders that make notches in the rod is likely to decrease the resistance of rod to cyclic loading and should be avoided. Additionally, the choice of rod material and diameter has an influence on the ability to bend it, its stiffness and its resistance to cyclic load. Manual force is exerted on the rod until it is securely fixed to two or three anchors above the osteotomy. This strategy reduces the risk of screw pullout, which is possible if a single screw is loaded. In difficult cases, a sublaminar-band-assisted technique for osteotomy closure can be used [24]. During closure of the osteotomy attention is paid to potential impingement of the dural sac or roots by bony edges or ligament remnants. Some dural kinking is common and can be resolved by sufficient undercutting of the laminae. A C-arm lateral view with two parallel images at $\mathrm{S} 1$ and $\mathrm{L} 1$ is used to measure the final lordosis. If the general condition of the patient is not critical, any undercorrection must be addressed to obtain the planned alignment (generally expressed as magnitude of lumbar lordosis). Ideally, posterior interlaminar contact at the end of the closure of the osteotomy should be achieved. This 

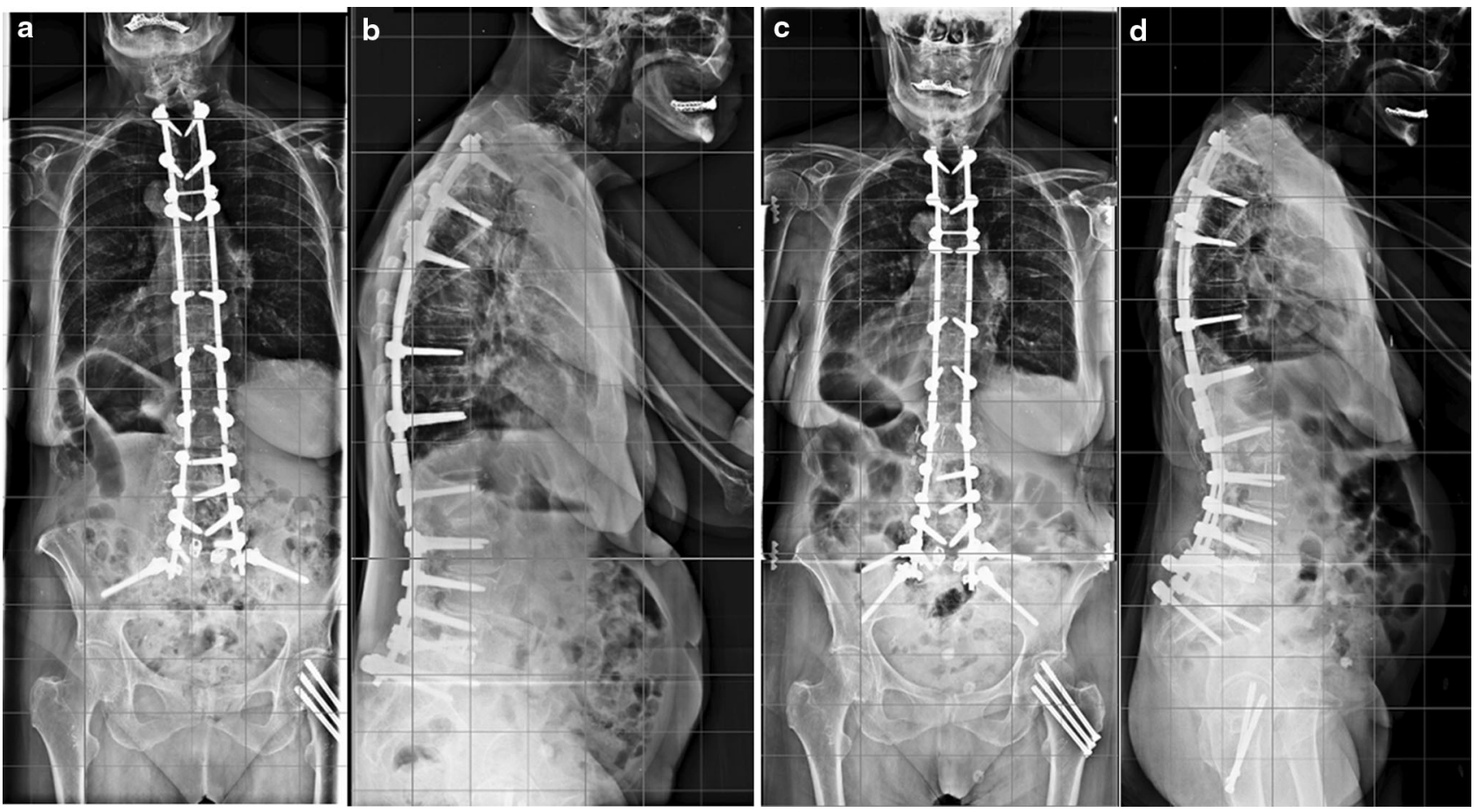

Fig. 5 a, b AP and lateral X-rays of a 68-year-old female with a rod breakage after a T5-pelvis fusion with L4 PSO. c, d Rod substitution after L3-4 and L4-5 intersomatic cage implant through XLIF

increases the stability and the chance of obtaining a solid fusion. After closure of the osteotomy evoked potentials are run to detect damage to neural structures. Meticulous preparation of a posterior fusion bed along the instrumented area, plus intertransverse fusion bed around the osteotomy is essential to obtain fusion. Grafting is performed with local bone, eventually supplemented with iliac crest, homologous bone or other bone substitutes.

\section{Postoperative care}

Postoperatively the patient is usually kept in intensive care for $24 \mathrm{~h}$. After $48-72 \mathrm{~h}$, they can sit and stand up. Long, full spine standing $\mathrm{X}$-rays are obtained as soon as the patient can stand without assistance to assess the final alignment. A thoracolumbar orthosis, to decrease cyclic load on the rods, worn for 4 months during the daytime is appropriate if tolerated.

A satisfactory postoperative alignment should include a SVA less than $5 \mathrm{~cm}$ from the posterosuperior corner of the sacrum and a PT not exceeding $21^{\circ}$, as these thresholds have demonstrated to be related to improved outcomes. Postoperative alignment resulting in imbalance has shown to be associated with increased risk of reoperation and poorer clinical outcomes in terms of VAS and ODI score reduction, and can be a risk factor for implant failure [25]. approach. This procedure reduces the risk of pseudoarthrosis increasing the stiffness of the anterior column

A thoracolumbar orthosis worn for 4 months during the daytime is appropriate, if tolerated to decrease cyclic load on the rods and can protect to some extent from the risk of rod failure. Our rehabilitation protocol consists mainly in instruction for independent ambulation and isometric exercises for the abdominal and back muscles in supine position, and change of position from standing to sitting and laying. No attempt is made to increase the range of motion of the spine in the postoperative period.

\section{Discussion}

Two key issues for clinicians treating adult spine deformity (ASD) are: complications and beneficial outcome for patients after surgery.

The fate of the distal discs - when the lower instrumented vertebra is L5-is a concern for surgeons. Cho et al. [26] compared fusion ending at L5 versus ending at S1 in adults with scoliosis. Blood loss and operative complications were similar between the two groups. Correction of the lumbar lordosis was significantly better in the $\mathrm{S} 1$ group. After 2 years of follow-up, $58 \%$ of the patients in the L5 group developed significant degenerative disc disease at L5-S1; half of them were symptomatic. The authors recommend that fusion to $\mathrm{S} 1$ is preferable to fusion to L5, even in patients with a normal L5-S1 segment of motion. 
Mechanical and general complications are frequent in surgery for ASD. In 2011, the Scoliosis Research Society (SRS) published a large study including nearly 5,000 cases of surgery for adult scoliosis reported in a society database [27]. They found an overall complication rate of $13.4 \%$, without significant differences due to age group (over and below 60 years) or etiology (idiopathic vs degenerative). Higher complication rate was observed in revisions, anterior and posterior approaches and osteotomies. The reported mortality rate was $0.3 \%$.

A different study from the SRS with focus on patients with rigid sagittal deformity undergoing surgery presents different data [28]. This study includes 578 patients. Twenty-nine percent of them had complications, and the rate was higher in cases requiring osteotomies $(34.8 \%)$. There was an increase in complication rates from no osteotomy (17.0 \%), to SPO (28.1\%), to PSO (39.1\%), to VCR $(61.1 \%)$. Raw mortality was $0.5 \%$, and it was not related to the type of osteotomy or even patients without osteotomies. In this study, patient's age was not related with different risk of complications.

A systematic review of the literature on complications for ASD surgery in patients older than 60 years [29] found a complication rate of $38 \%$ and $0.85 \%$ of mortality. The same study found substantial improvements in outcomes measures in these patients, with average ODI improvement of $24 \%$ and VAS improvement of 5.2 points in a ten-point scale. There was no specific information regarding procedures with osteotomies. Smith et al. [15] in a multicenter study that retrospectively reviewed a prospectively built database with 2-year follow-up of patients operated for ASD reported a complication rate that increased with age group from $17 \%$ (below 45 years) to $42 \%$ (45-64 years), and $71 \%$ (older than 65 years). The clinical outcomes measured with ODI and VAS scores were similar across age groups, but the baseline status was worse as age increased. Thus, older patients had higher complication rates but also higher clinical benefit as shown by these outcomes measures. There were no deaths reported in this series of 453 patients.

\section{Conclusions}

PSO is a valuable surgical procedure in correction of severe hypolordosis in the lumbar spine. It is a demanding procedure for the surgeon, the anesthesiologist and the rehabilitation team. Key factors of success are correct indication (considering the clinical impact of the deformity, the general condition of the patient, the degree of the deformity and the experience of the hospital team), appropriate surgical planning with correct calculation of the correction needed, diligent execution of the procedure and high-level anesthesiological and medical care. In spite of high complication rates, it has a substantial positive impact in the quality of life of patients, including the elderly.

Some excellent surgical videos describing different modalities of the PSO technique have been published in the ESJ's OOT film collection (www.oot-esj.com) and we recommend to view the films [24, 30-32].

\section{Conflict of interest None.}

Open Access This article is distributed under the terms of the Creative Commons Attribution License which permits any use, distribution, and reproduction in any medium, provided the original author(s) and the source are credited.

\section{References}

1. Bridwell KH, Baldus C, Berven S, Edwards C 2nd, Glassman S, Hamill C, Horton W, Lenke LG, Ondra S, Schwab F, Shaffrey C, Wootten D (2010) Changes in radiographic and clinical outcomes with primary treatment adult spinal deformity surgeries from two years to three- to five-years follow-up. Spine 35(20):1849-1854

2. Schwab FJ, Lafage V, Farcy JP, Bridwell KH, Glassman S, Shainline MR (2008) Predicting outcome and complications in the surgical treatment of adult scoliosis. Spine 33(20):2243-2247

3. Glassman SD, Berven S, Bridwell K, Horton W, Dimar JR (2005) Correlation of radiographic parameters and clinical symptoms in adult scoliosis. Spine 30(6):682-688

4. Terran J, Schwab F, Shaffrey CI, Smith JS, Devos P, Ames CP, Fu KM, Burton D, Hostin R, Klineberg E, Gupta M, Deviren V, Mundis G, Hart R, Bess S, Lafage V, International Spine Study Group (2013) The SRS-Schwab adult spinal deformity classification: assessment and clinical correlations based on a prospective operative and nonoperative cohort. Neurosurgery 73(4):559-568

5. Berjano P, Lamartina C (2014) Classification of degenerative segment disease in adults with deformity of the lumbar or thoracolumbar spine. Eur Spine J 23(9):1815-1824

6. Lamartina C, Berjano P (2014) Classification of sagittal imbalance based on spinal alignment and compensatory mechanisms. Eur Spine J 23(6):1177-1189

7. Schwab F, Lafage V, Patel A, Farcy JP (2009) Sagittal plane considerations and the pelvis in the adult patient. Spine 34(17): 1828-1833

8. Schwab F, Patel A, Ungar B, Farcy JP, Lafage V (2010) Adult spinal deformity-postoperative standing imbalance: how much can you tolerate? An overview of key parameters in assessing alignment and planning corrective surgery. Spine 35(25):2224-2231

9. Lamartina C, Berjano P, Petruzzi M, Sinigaglia A, Casero G, Cecchinato R, Damilano M, Bassani R (2012) Criteria to restore the sagittal balance in deformity and degenerative spondylolisthesis. Eur Spine J 21S(1):S27-S31

10. Lafage V, Schwab F, Vira S, Patel A, Ungar B, Farcy JP (2011) Spinopelvic parameters after surgery can be predicted. Spine 36:1037-1045

11. Berjano P, Lamartina C (2013) Far lateral approaches (XLIF) in adult scoliosis. Eur Spine J 22S(2):S242-S253

12. Smith-Petersen MN, Larson CB, Aufranc OE (1945) Osteotomy of the spine for correction of flexion deformity in rheumatoid arthritis. J Bone Joint Surg 27:1-11 
13. Ponte A, Vero B, Siccardi GL (eds) (1984) Surgical Treatment of Scheuermann's Hyperkyphosis. Aulo Gaggi, Bologna

14. Berjano P, Langella F, Ismael MF, Damilano M, Scopetta S, Lamartina C (2014) Successful correction of sagittal imbalance can be calculated on the basis of pelvic incidence and age. Eur Spine J 23(S6):587-596

15. Smith JS, Shaffrey CI, Glassman SD, Berven SH, Schwab FJ, Hamill CL, Horton WC, Ondra SL, Sansur CA, Bridwell KH, Spinal Deformity Study Group (2011) Risk-benefit assessment of surgery for adult scoliosis: an analysis based on patient age. Spine 36(10):817-824

16. Le Huec JC, Leijssen P, Duarte M, Aunoble S (2011) Thoracolumbar imbalance analysis for osteotomy planification using a new method: FBI technique. Eur Spine J 20(S5):669-680

17. Berjano P, Cecchinato R, Damilano M, Morselli C, Sansone V, Lamartina C (2013) Preoperative calculation of the necessary correction in sagittal imbalance surgery: validation of three predictive methods. Eur Spine J 22S(6):S847-S852

18. Berjano P, Damilano M, Bozzaro M, Pejrona M, Cecchinato R, Lamartina C (2013) Standing lateral lumbar spine and pelvis (SLLP) radiograph: a screening, reduced radiation method, for sagittal imbalance. Eur Spine J 22(S6):S842-S846

19. Aurouer N, Obeid I, Gille O, Pointillart V, Vital JM (2009) Computerized preoperative planning for correction of sagittal deformity of the spine. Surg Radiol Anat 31(10):781-792

20. Lafage V, Schwab F, Vira S, Hart R, Burton D, Smith JS, Boachie-Adjei O, Shelokov A, Hostin R, Shaffrey CI, Gupta M, Akbarnia BA, Bess S, Farcy JP (2011) Does vertebral level of pedicle subtraction osteotomy correlate with degree of spinopelvic parameter correction? J Neurosurg Spine 14:184-191

21. Koller H, Acosta F, Zenner J, Ferraris L, Hitzl W, Meier O, Ondra S, Koski T, Schmidt R (2010) Spinal surgery in patients with Parkinson's disease: experiences with the challenges posed by sagittal imbalance and the Parkinson's spine. Eur Spine J 19(10):1785-1794

22. Bissolotti L, Gobbo M, Zaina F, Lusini M, Donzelli S, Negrini S (2013) Scoliosis and sagittal balance in Parkinson's disease: analysis of correlations. Scoliosis 8(Suppl 2):O7
23. Enercan M, Ozturk C, Karaman S, Sarier M, Hamzaoglu A, Alanay A (2012) Osteotomies/spinal column resections in adult deformity. Eur Spine J 22(2 suppl):254-264

24. Berjano P, Cucciati L, Damilano M, Pejrona M, Lamartina C (2013) A novel technique for sublaminar-band-assisted closure of pedicle subtraction osteotomy. Eur Spine J 22(12):2910-2914

25. Berjano P, Bassani R, Casero G, Sinigaglia A, Cecchinato R, Lamartina C (2013) Failures and revisions in surgery for sagittal imbalance: analysis of factors influencing failure. Eur Spine $\mathbf{J}$ 22S(6):S853-S858

26. Cho K-J, Suk S-I, Park S-R, Kim J-H, Choi S-W, Yoon Y-H, Won M-H (2009) Arthrodesis to L5 versus S1 in long instrumentation and fusion for degenerative lumbar scoliosis. Eur Spine J 18:531-537

27. Sansur CA, Smith JS, Coe JD, Glassman SD, Berven SH, Polly DW Jr, Perra JH, Boachie-Adjei O, Shaffrey CI (2011) Scoliosis research society morbidity and mortality of adult scoliosis surgery. Spine 36(9):E593-E597

28. Smith JS, Sansur CA, Donaldson WF 3rd, Perra JH, Mudiyam R, Choma TJ, Zeller RD, Knapp DR Jr, Noordeen HH, Berven SH, Goytan MJ, Boachie-Adjei O, Shaffrey CI (2011) Short-term morbidity and mortality associated with correction of thoracolumbar fixed sagittal plane deformity: a report from the Scoliosis Research Society Morbidity and Mortality Committee. Spine 36(12):958-964

29. Drazin D, Shirzadi A, Rosner J, Eboli P, Safee M, Baron EM, Liu JC, Acosta FL Jr (2011) Complications and outcomes after spinal deformity surgery in the elderly: review of the existing literature and future directions. Neurosurg Focus 31(4):E3. doi:10.3171/ 2011.7.FOCUS11145

30. Obeid I, Bourghli A, Vital JM (2012) Pedicle subtraction osteotomy for postoperative flat back and sagittal imbalance. Eur Spine J 21(6):1218-1219

31. Le Huec JC, Aunoble S (2012) Pedicle subtraction osteotomy for sagittal imbalance. Eur Spine J 21(9):1896-1897

32. Hedlund R (2012) Pedicle subtraction osteotomy in flat back syndrome 38 years after Harrington instrumentation for AIS. Eur Spine J 21(3):563-565 